\title{
Aus einem Schreiben des Herrn Schubert an Herrn Prof. Winlock.
}

Wheu the Observations of Eunomia, made in 1860 , were published, I was struck with the difference of 4 Seconds in time in $\alpha$ from my Ephemeris.

A mistake in the computation of the elements was found: I had used Callet's 'Tables of 7 figures; in those Tables the change of the first three figures of the mantissa is made rather awkward, and 1 had put down $\log \frac{d y}{d t}=$ 7,0512706 nistead of 7,0522706 . The correct osculating elements are now :
1860 Jan. 1,0 Washington M. T.

$$
\left.\begin{array}{rl}
\boldsymbol{M} & =265^{\circ} 14^{\prime} 17^{\prime \prime} 5 \\
\pi & =273120,2 \\
\Omega & =2935648,5 \\
\boldsymbol{i} & =114341,4
\end{array}\right\} \text { M. Equin. Ep. }
$$

These elements with the perturbations by Jupiter and Saturn since 1860 Jan. 1,0 give now for the normal-place 1860 Aug. 24,5

$$
\begin{array}{cc}
\Delta \alpha & \Delta \delta \\
+1^{s} 34 & +13^{\prime \prime} 4
\end{array}
$$

Eunomia came very near the Earth in that 0pposition and the error of the elements appears, therefore, greater than in 1858 and 1859 .

Ann Arbor 1861 0et. 15.

E. Schubert.

Elemente und Oppositionsephemeride von (59) Elpis, von Herrn Dr. E. Weiss.

Mitgetheilt von Herrn Professor, Director von Littrow.

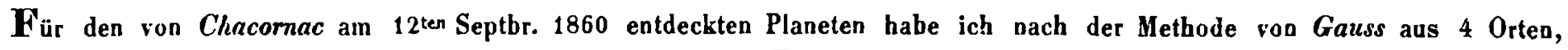
welche einen Zeitraum von 144 Tagen umfassen, folgendes Elementensystem erhalten:

$$
\begin{array}{rl}
M & 1862 \text { Januar } 0,0 \\
\pi & =92^{\circ} 32^{\prime} 40^{\prime \prime} 3 \\
\pi & =17443,2 \\
\Omega & =1702158,8 \\
i & =83753,4 \\
\varphi & =6459,5 \\
\log a & =0,4337160 \\
\log e & =9,0703446 \\
\mu & =793^{\prime \prime 2} 2911
\end{array}
$$

Ephemeride für dic oppositionvon Scheinbare

$12^{\text {h }}$ mittl. Berl. Zt.

1861 Jan. 12

13
14
15
16
17
18
19
20
21
22
23

Diese Bahn stellt, wie die Vergleichung einer Anzahl von Beobachtungen zeigte, die ganze Beobachtungsreihe befriedigend dar. Ich habe daher nach diesen Elementen die folgende Ephemeride berechnet, und glaube, dass sie den Ort des Planeten sehr nahe angeben wird. 